\title{
Thin-sheet electromagnetic inversion modeling using Monte Carlo Markov Chain (MCMC) algorithm
}

\author{
Hendra Grandis ${ }^{1}$, Michel Menvielle ${ }^{2}$, and Michel Roussignol ${ }^{3}$ \\ ${ }^{1}$ Department of Geophysics and Meteorology, Institut Teknologi Bandung (ITB), Jalan Ganesha 10, Bandung - 40132, Indonesia \\ ${ }^{2}$ Centre d'Etude des Environnements Terrestre et Planétaires, 3, Avenue de Neptune, F-94107 Saint Maur des Fosses, France \\ ${ }^{3}$ Equippe d'Analyse et de Mathématique Appliquée, Université de Marne la Vallée, 5, Boulevard Descartes, F-77454 Marne la Vallée, France
}

(Received November 20, 2000; Revised February 13, 2002; Accepted February 13, 2002)

\begin{abstract}
The well-known thin-sheet modeling has become a very useful interpretation tool in electromagnetic (EM) methods. The thin-sheet model approximates fairly well 3-D heterogeneities having a limited vertical dimension. This type of approximation leads to amenable computation of EM response of a relatively complex conductivity distribution. This paper describes the integration of thin-sheet forward modeling into an inversion method based on a stochastic Monte Carlo Markov Chain (MCMC) algorithm. Effective exploration of the model space is performed using a biased sampler capable to avoid entrapment to local minima frequently encountered in a such highly nonlinear problem. Results from inversion of synthetic EM data show that the algorithm can reasonably resolve the true structure. Effectiveness and limitations of the proposed inversion method is discussed with reference to the synthetic data inversions.
\end{abstract}

\section{Introduction}

The thin-sheet modeling has proven to be an effective tool for the interpretation of electromagnetic (EM) data, especially when heterogeneities are confined in a layer having limited vertical dimension. The thin-sheet approximation is valid if the thickness of the thin layer containing heterogeneities is much smaller than the penetration depth of EM fields. The model is then represented by lateral variations of conductance, i.e. integrated conductivity over the thickness of the thin layer. This approximation significantly simplifies the resolution of the Maxwell's equations describing the EM fields in quasi three-dimensional (3-D) media. There are several working algorithms employing integral equation method for thin-sheet EM modeling (e.g. Vasseur and Weidelt, 1977; McKirdy et al., 1985) and their advanced versions as reported by Wang and Lilley (1999, and references therein).

An inversion scheme devised to resolve strongly nonlinear problems, such as those in EM methods, has been recently proposed by Grandis et al. (1999, 2002). The inverse problem recast in the Bayesian inference approach is solved by using a stochastic Monte Carlo Markov Chain (MCMC) algorithm. The method has been successfully applied to 1-D magnetotelluric (MT) modeling for both synthetic and real data as well (Grandis, 1997; Grandis et al., 1999). Similar approach applied to thin-sheet EM modeling also gave encouraging results (Jouanne, 1991; Roussignol et al., 1993). In the latter, Markov chains were used not only to update the model but also to estimate the electric field as iteration pro-

Copy right(c) The Society of Geomagnetism and Earth, Planetary and Space Sciences (SGEPSS); The Seismological Society of Japan; The Volcanological Society of Japan; The Geodetic Society of Japan; The Japanese Society for Planetary Sciences. gresses. The convergence of the modified (or 2-D) Markov chain has been proven empirically only for cases in which a resistive heterogeneity is contained in a more conductive host layer.

We have incorporated the thin-sheet forward modeling scheme of Vasseur and Weidelt (1977) in our inversion algorithm for its simplicity in handling variable exchange (input and output) between forward and inverse part of the algorithm. We will first describe the inversion algorithm and also outline the approach adopted by Jouanne (1991) and Roussignol et al. (1993). Then, a modification of the algorithm is proposed in order to overcome difficulties encountered in the previous approach. The modification consists in a quasi-complete resolution of the electric field involved in the forward modeling calculation. The method was tested to invert synthetic data corresponding to simple resistive (or conductive) structure in a conductive (or resistive) host. A special attention has been paid to the resolving capability of the method faced to particularities of EM data (i.e. MT impedance tensor and induction vector) and also to different discretization of prior conductance.

\section{MCMC Inversion Algorithm}

For completeness, we will describe the non-linear inversion method based on MCMC algorithm focusing mainly on practical aspects of the method in order to illustrate more clearly how the algorithm works. The readers are referred to Grandis et al. (1999, 2002, and references therein) for theoretical details of the method.

In the Bayesian perspective, resolving an inverse problem can be stated as updating our a priori beliefs on the model by using information acquired from observations which results in a posteriori knowledge on the model sought. The 
Bayesian approach naturally integrates uncertainties of the information involved by using probability density function (pdf) representation (Robert, 1992). For discrete (or discretized) quantities appropriate for our problem, the Bayesian formula takes the following form

$$
p(\mathbf{m} \mid \mathbf{d})=\frac{g(\mathbf{d} \mid \mathbf{m}) h(\mathbf{m})}{\sum_{\mathbf{m} \in E} g(\mathbf{d} \mid \mathbf{m}) h(\mathbf{m})}
$$

where $\mathbf{d}$ and $\mathbf{m}$ denote data and model vector respectively. In Eq. (1), the posterior probability is represented by the conditional probability for the model given the data $p(\mathbf{m} \mid \mathbf{d})$ which in fact is the solution to the inverse problem. The conditional probability of the data for a given model $g(\mathbf{d} \mid \mathbf{m})$ combines both statistics of the data and the resolution of the forward problem while $h(\mathbf{m})$ is the probability of the prior model. We are usually more interested in the probability for each (or for a certain) model parameter regardless of other model parameters. This marginal posterior probability of a model parameter $m_{i}$ is obtained from Eq. (1) by taking integral (or sum in our discrete case) over other model parameters $m_{j \neq i}$ such that

$$
p\left(m_{i} \mid \mathbf{d}\right)=\frac{\sum_{m_{j \neq i}} g(\mathbf{d} \mid \mathbf{m}) h(\mathbf{m})}{\sum_{\mathbf{m} \in E} g(\mathbf{d} \mid \mathbf{m}) h(\mathbf{m})} .
$$

Significance of this marginal posterior probability of a model parameter will be explained in the subsequent paragraphs.

The denominator of Eqs. (1) and (2) is in fact a normalizing constant and it sums over the entire possible models in the model space. For a model consisting of $M$ model parameters, i.e. $\mathbf{m}=\left[m_{i}\right](i=1,2, \ldots, M)$, and the $i$-th model parameter can take $N_{i}$ discrete possible (prior) values $\rho_{j}\left(j=1,2, \ldots, N_{i}\right)$ then the number of possible models in $E$ is the product of $N_{1} \times N_{2} \times \cdots \times N_{M}$. For a homogeneous parameterization used in our case the number of possible (prior) values is the same for each model parameter. Then the number of possible models in $E$ is $N^{M}$. However in any case, numerical computation of (1) or (2) is impractical or even impossible for most geophysical problems having a large number of model parameters and complicated non-linear forward problem. A stochastic algorithm is then formulated to efficiently sample the model space. Unlike pure Monte Carlo methods which sample randomly the model space with uniform probabilities, the Gibbs sampler used in the algorithm employs a certain conditional probability to bias the sampling process such that regions having significant contribution to the posterior pdf are sampled efficiently.

Consider a case in which model parameters other than $m_{i}$ are fixed to their actual (or most recent) value. Then, we have a conditional posterior probability for model parameter $m_{i}$ given other model parameters $m_{j \neq i}$ fixed, i.e.

$$
\hat{p}\left(m_{i}=\rho_{k} \mid \mathbf{d}\right)=\frac{g\left(\mathbf{d} \mid m_{i}=\rho_{k}\right) h\left(m_{i}=\rho_{i}\right)}{\sum_{l=1}^{N} g\left(\mathbf{d} \mid m_{i}=\rho_{l}\right) h\left(m_{i}=\rho_{l}\right)} ;
$$

In the above equation we explicitly state that $m_{i}$ can take $\rho_{k} ; k=1,2, \ldots, N$ as its value such that $\hat{p}\left(m_{i}=\rho_{k}\right.$ । d) $\equiv f\left(\rho_{k}\right)$, which is also applicable for $p\left(m_{i} \mid \mathbf{d}\right)$. The difference is that in Eq. (2) we have to consider all possible values for $m_{j \neq i}$ and sum the probabilities associated to them, while in Eq. (3) only probabilities related to actual values for $m_{j \neq i}$ are concerned. The normalizing constant in Eq. (3) involves a sum over all possible values for the model parameters such that it can be amenable for a reasonable number of $N$.

The importance of the conditional posterior probability for a model parameter given in Eq. (3) will be evident by making it more explicit. By establishing $\mathbf{d}=\left[d_{i}\right] i=$ $1,2, \ldots, N D$ as observational data, then $g(\mathbf{d} \mid \mathbf{m})$ is commonly called likelihood function (Jackson and Matsu'ura, 1985; Sen and Stoffa, 1996). We consider that data errors are independent and obey a Gaussian distribution with zero mean and variance $\sigma^{2}$ so that the likelihood function can be written as

$$
g(\mathbf{d} \mid \mathbf{m})=C \exp \left(-\frac{1}{2} \sum_{j=1}^{N D}\left(\frac{d_{j}-[F(\mathbf{m})]_{j}}{\sigma_{j}}\right)^{2}\right)
$$

where $[F(\mathbf{m})]_{j}$ is the $j$-th component of a vector resulting from an application of the forward modeling operator $F$ and $C$ retains all constants involved. Substituting Eq. (4) to Eq. (3) by further assuming a homogeneous probability for the prior model results in a more explicit formula as follows

$$
\begin{aligned}
\hat{p}\left(m_{i}=\right. & \left.\rho_{k} \mid \mathbf{d}\right) \\
= & C \exp \left(-\frac{1}{2} \sum_{j=1}^{N D}\left(\frac{d_{j}-\left[F\left(m_{i}=\rho_{k}\right)\right]_{j}}{\sigma_{j}}\right)^{2}\right) ; \\
& k=1,2, \ldots, N
\end{aligned}
$$

where $C$ again absorbs all normalizing constants including the denominator of Eq. (3). For a fixed $m_{j \neq i}$ Eq. (5) represents relative probabilities of $\rho_{k}(k=1,2, \ldots, N)$ as possible values for $m_{i}$. Thus, Eq. (5) can be utilized to update $m_{i}$ by using a value drawn from $\rho_{k}$ weighted by $\hat{p}\left(m_{i}=\rho_{k} \mid \mathbf{d}\right) ; k=1,2, \ldots, N$. It's obvious that $\rho_{k}$ corresponding to a smaller misfit will have a greater probability to be selected. However, values with greater misfit still have a chance to be selected, they are only less probable. The latter gives the algorithm an ability to escape local minima frequently encountered in strongly non-linear inverse problems. Figure 1 illustrates the model parameterization used in the Markov chain algorithm and how the conditional posterior probability is used to update a model parameter.

Updating model parameters randomly or sequentially generates a series of states (or models) obeying Markov chain rules, i.e. probability of a state depends on the past states only through the previous (or immediate past) state. The iterative process leads to a Markov chain in a set of finite possible models $E$ and is commonly termed Gibbs sampler (Robert, 1996). The conditional posterior probability (5) serves as the transition probability that governs the evolution in time or iterations of the Markov chain. In fact, the conditional posterior probability is equal to the transition probability of the Markov chain up to a constant multiplier (Grandis et al., 2002). Fundamental properties of a similar Markov chain, especially its asymptotic behaviors, 
(a)

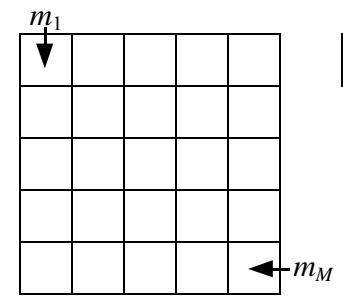

(b)

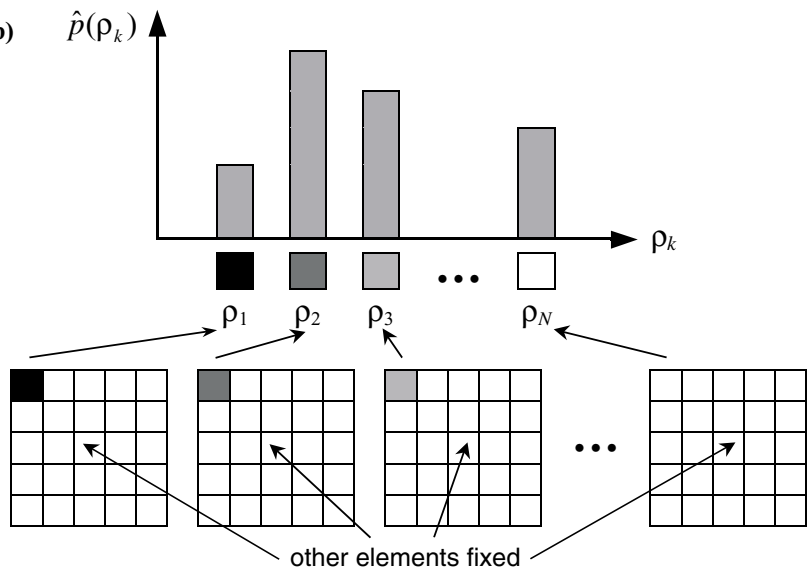

Fig. 1. A schematic diagram illustrating (a) model parameterization and (b) selection of prior values to update a parameter model using the conditional posterior probability.

have been discussed in detail by Rothman (1986). Here, we only exploit their practical consequences. After sufficiently a long time the Markov chain exhibits a stationary state - independent of its initial state - described by its invariant probability. It has been shown (see e.g. Grandis et $a l ., 1999,2002)$ that the invariant probability of the constructed Markov chain is in fact the posterior probability defined in Eq. (1). Further theorem on asymptotic behaviors of the Markov chain implies that in general empirical averages tend towards statistical averages (Heerman, 1990; Robert, 1996). This in turn simplifies the evaluation of posterior quantities, especially the marginal posterior probability from which statistical measures for a model parameter (mean and variance) can be estimated.

\section{Thin-Sheet Inversion Algorithm}

In the thin-sheet modeling, calculation of the electric field is performed by discretizing the thin layer containing heterogeneities into rectangular uniform cells. The conductance of each cell is assumed constant. By using the integral equation method, the computation domain covers only the anomalous zone where the conductance differs from that of normal host medium. The electric field is then used to calculate the magnetic field to obtain theoretical or calculated EM transfer functions (i.e. MT impedance tensor and magnetic transfer function).

The total electric field in the $k$-th cell located at the heterogeneous layer is expressed by (Vasseur and Weidelt, 1977)

$$
\mathbf{E}(k)=\mathbf{E}^{n}(k)-i \omega \mu_{0} \sum_{k \in K} \tau_{k^{\prime}} \mathbf{G}\left(k, k^{\prime}\right) \cdot \mathbf{E}\left(k^{\prime}\right),
$$

where $\mathbf{E}^{n}(k)$ is the normal electric field associated with nor- mal (stratified or 1-D) conductivity distribution, $\mathbf{G}\left(k, k^{\prime}\right)$ is the Green kernel representing electric field at $k$ due to a unitary dipole at $k^{\prime}$ and $\tau_{k}$ denotes anomalous conductance of the $k$-th cell, $k, k^{\prime} \in K$. Equation (6) is in fact a system of $N=2 K$ linear equations associated to orthogonal electric fields $\left(E_{x}, E_{y}\right)$ at each cell corresponding to a certain conductance distribution. However, in the subsequent equations the vector representation of the electric field is retained for the sake of clarity.

For a large number of cells in the anomalous domain, a direct matrix inversion to resolve (6) is prone to round-off errors and instability and may require a considerable computer memory. Additionally, the form of Eq. (6) is such that it is more suitable to use the iterative Gauss-Seidell method in which an initial value of the solution is updated iteratively to obtain the solution (Jain et al., 1987). A particular characteristic of the Gauss-Seidell method is the use of updated elements of the solution to estimate the remaining elements. At $(j+1)$-th iteration, the estimated electric field is calculated by

$$
\begin{aligned}
\hat{\mathbf{E}}(k)_{j+1}= & \frac{1}{1+i \omega \mu_{0} \tau_{k} \mathbf{G}(k, k)} \\
& \times\left(\mathbf{E}^{n}(k)-i \omega \mu_{0}\left[\sum_{k^{\prime}=1}^{k-1} \tau_{k^{\prime}} \mathbf{G}\left(k, k^{\prime}\right) \cdot \mathbf{E}\left(k^{\prime}\right)_{j+1}\right.\right. \\
& \left.\left.\quad+\sum_{k^{\prime}=k+1}^{K} \tau_{k} \mathbf{G}\left(k, k^{\prime}\right) \cdot \mathbf{E}\left(k^{\prime}\right)_{j}\right]\right)
\end{aligned}
$$

where the normal electric field is constant throughout the iteration process. To accelerate convergence we use an overrelaxation parameter $w$ such that

$$
\mathbf{E}(k)_{j+1}=\mathbf{E}(k)_{j}+w\left(\hat{\mathbf{E}}(k)_{j+1}-\mathbf{E}(k)_{j}\right) .
$$

The normal electric field $\mathbf{E}^{n}(k)$ is commonly used as the initial value for $\mathbf{E}(k)_{0}$ and in general convergent solution is obtained after 10 to 20 iterations, as long as the conductivity contrast is not too high (Vasseur and Weidelt, 1977).

In order to reduce the computation time of the forward modeling, Jouanne (1991) and Roussignol et al. (1993) adopted a 2-D Markov chain approach to simultaneously estimate the conductance and the electric field of a cell. At a given step (i.e. conductance change of the $k$-th cell), the electric field at $k$-th cell is computed by performing one (incomplete) Gauss-Seidell iteration based on previous step values using Eqs. (7) and (8). The electric field in all other cells different than $k$ is computed to account for the change of conductance and electric field in the $k$-th cell by using

$$
\mathbf{E}\left(k^{\prime}\right)_{j+1}=\mathbf{E}\left(k^{\prime}\right)_{j}+\left[\left(\tau_{k}\right)_{j+1}-\left(\tau_{k}\right)_{j}\right] \mathbf{E}(k)_{j+1}
$$

where $k^{\prime} \neq k$. This is an approximation that neglects secondary effect at cells different than $k$ and $k^{\prime}$ produced by changes of electric field and conductance in $k$-th cell. The pair $\left(\mathbf{m}_{j}, \mathbf{E}_{j}\right)$ constitutes, in effect, a Markov chain since it depends on their past states only through $\left(\mathbf{m}_{j-1}, \mathbf{E}_{j-1}\right)$. The 2-D Markov chain contains mixed variables, i.e. discrete (conductance) and continuous variable (electric field). Mathematical proof of convergence for such chain has not been established. However, empirical results using synthetic 

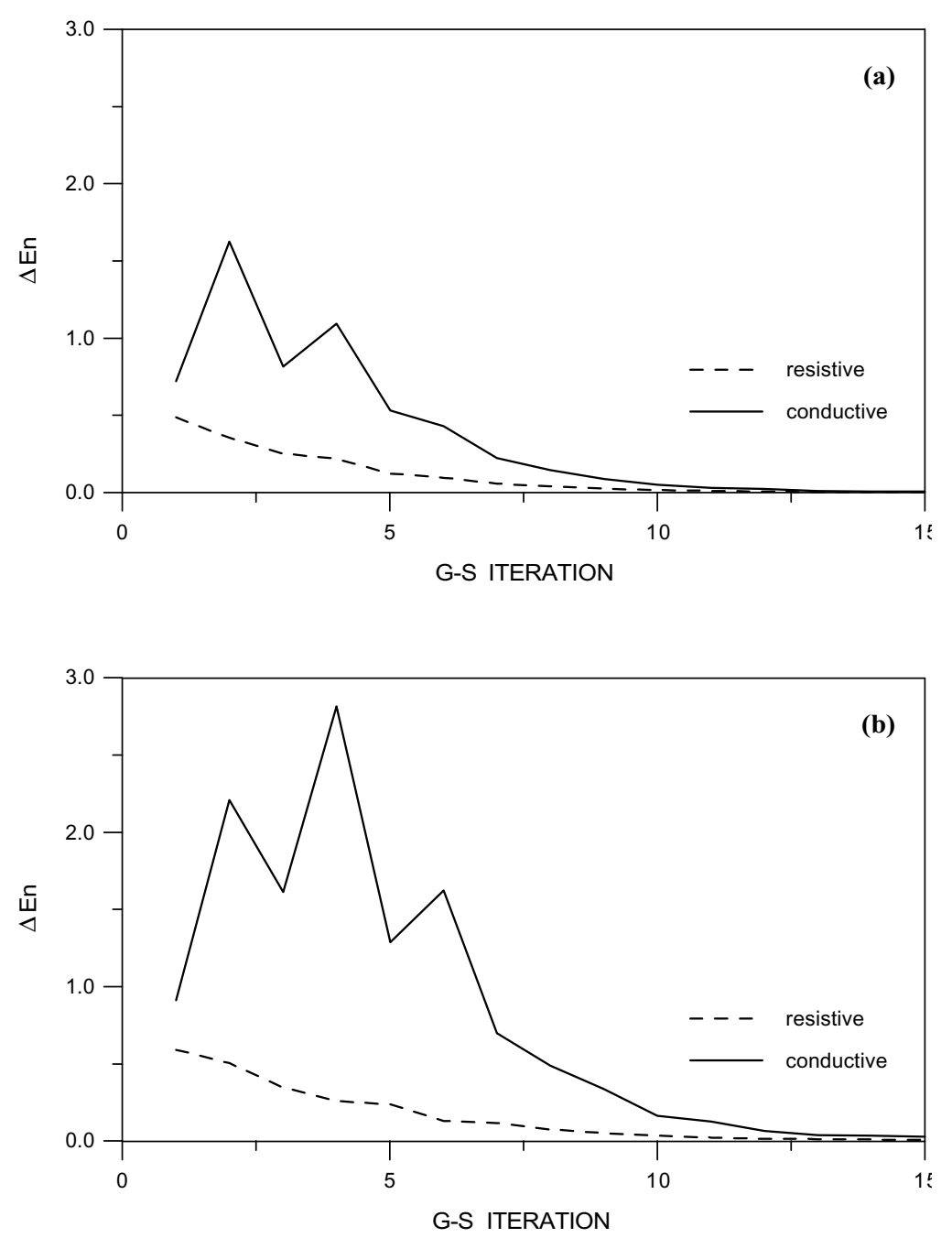

Fig. 2. Convergence curves of the electric field calculation corresponding to two different resistivity contrast between host and anomalous zone: (a) 10:500 Ohm.m and (b) 5:500 Ohm.m. It is shown that convergence is more difficult to attain and is slower for the conductive anomaly.

data showed that the chain is convergent for resistive heterogeneities in a conductive thin layer and the true or synthetic models were fairly well resolved. In the case where the heterogeneity is more conductive than the host medium, the Markov chain diverges (Jouanne, 1991; Roussignol et al., 1993). From practical point of view, the above facts exclude the application of inversion method using the Markov chain algorithm in the majority of interesting real situations where heterogeneities are mainly conductive in a more resistive environment. From theoretical point of view, empirical convergence of the Markov chain only for a certain class of models does not guarantee that it will be the case for every model in that class due to absence of mathematical proof. Therefore, convergence of the Markov chain must be established empirically for every class of models considered.

The assessment of the convergence of the forward modeling algorithm was performed by using synthetic models in order to identify possible causes of difficulties in resolving conductive anomalies in a resistive host. For a given conductance distribution in the thin-sheet and the stratified host medium, the resolution of the forward modeling is based on the calculation of the electric field at the thin-sheet. The convergence is attained when the difference of electric field estimates at two successive iterations tends to zero. A measure of convergence is expressed as a quadratic difference relative to the previous value and it is averaged over all grid points. In our cases the convergence is obtained in less than 20 iterations. Figure 2 presents convergence curves of the electric field computation for two resistivity ratios between the anomalous zone and the host medium (10:500 and 5:500 Ohm.m). The configuration (resistivity and thickness) of the stratified medium and the form of the anomalous zone is identical to the synthetic model for inversion described in the next section. It is obvious that other parameters also play important role in the convergence rate. However, it is evident from Fig. 2 that more Gauss-Seidell iterations in the forward modeling are needed to obtain convergence in cases where heterogeneities are conductive. The convergence curves even exhibit oscillating character in the first few iterations before monotonically decreasing to attain convergence. These results indicate that one incomplete Gauss-Seidell iteration and approximate electric field calculation as done by Jouanne (1991) and Roussignol et al. (1993) are inadequate to obtain accurate transition probabil- 


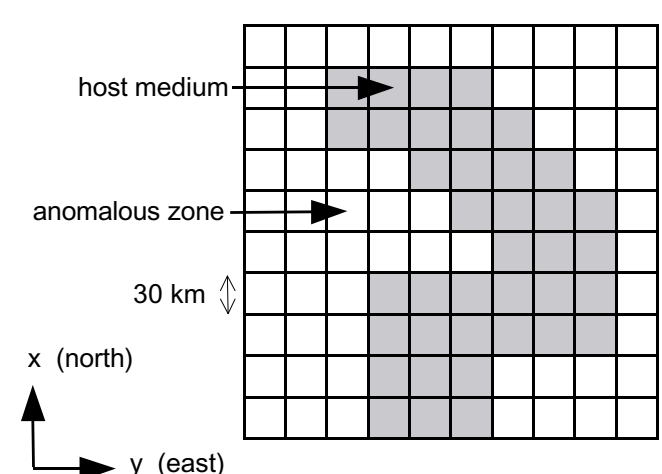

(a)

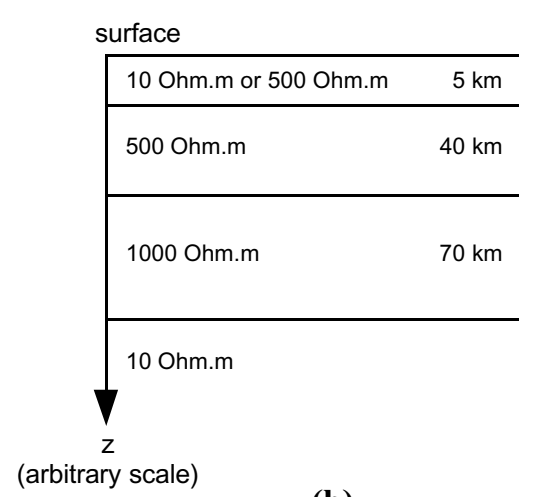

(b)

Fig. 3. Synthetic model: (a) geometry of the anomalous zone in the thin-sheet and (b) stratified or 1-D host medium containing the thin-sheet.

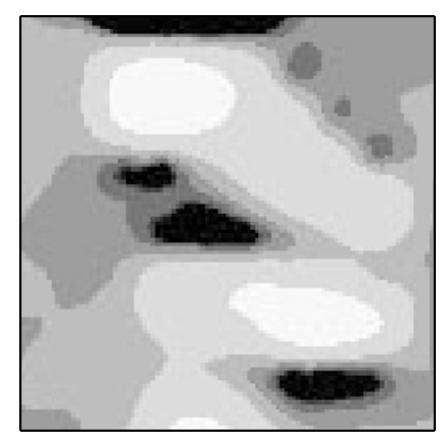

$\rho_{X Y}$

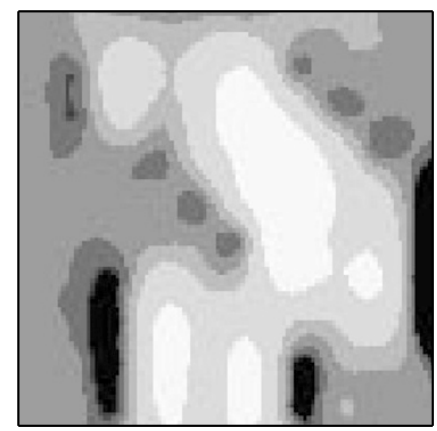

$\rho_{Y X}$

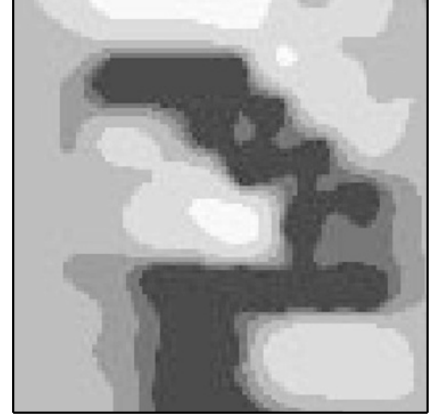

$\rho_{X Y}$

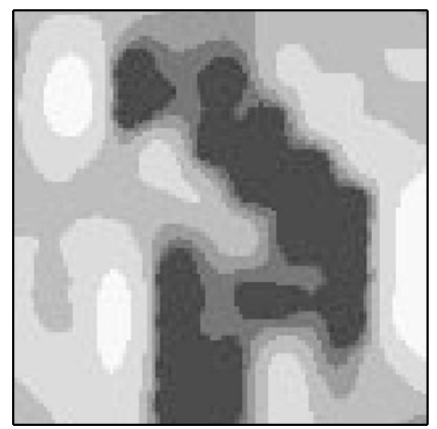

$\rho_{Y X}$

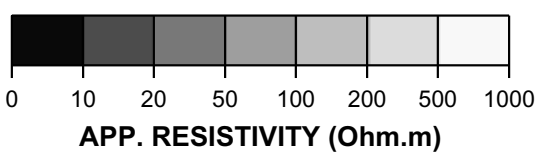

Fig. 4. Apparent resistivity maps calculated from principal components of the impedance tensor $\left(\rho_{x y}\right.$ and $\left.\rho_{y x}\right)$ for $T=1000$ sec., resistive anomaly (left) and conductive anomaly (right).

ity for the Markov chain. This in turn leads to convergence problem of the Markov chain especially in the case of conductive structure in a resistive host.

The Markov chain algorithm relies on the transition probability (5) such that if its precision is insufficient, it will lead to difficulty in generating convergent and optimal models. Therefore, we propose to modify the existing Markov chain algorithm to overcome the above limitations. The modifica- tion consists in performing several complete Gauss-Seidell iterations in the computation of the electric field (i.e. forward modeling) such that the computation of the transition probability (5) is sufficiently accurate. This will considerably increase the computation time of the algorithm. However, by using current electric field as the initial value, 2 or 3 Gauss-Seidell iterations were sufficient to obtain accurate transition probability (Grandis, 1994). This means that in 

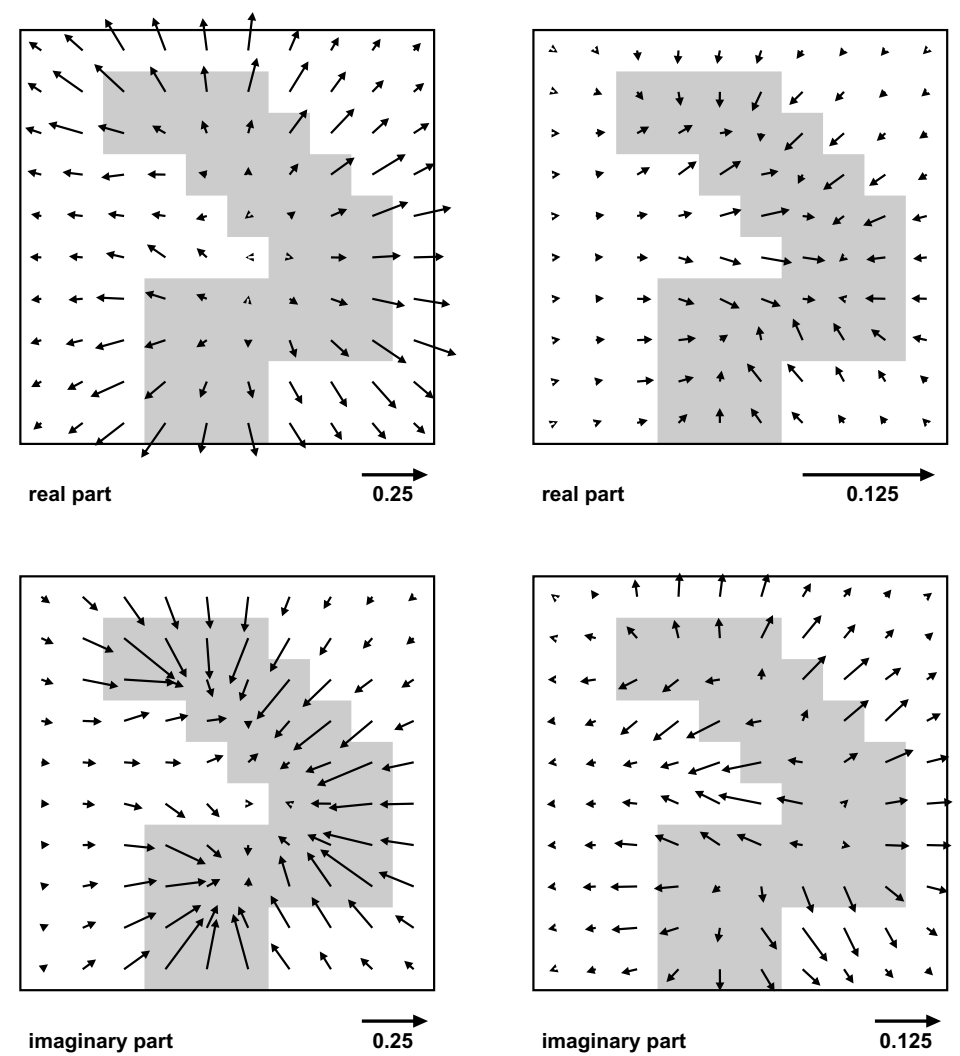

Fig. 5. Induction arrow map showing real and imaginary parts for $T=1000 \mathrm{sec}$. of the resistive anomaly (left) and the conductive anomaly (right).

fact, we need to resolve only partially the system of equations defining the electric field in the calculation of the transition probability. The Markov chain is supposed to be convergent in practically all situations (i.e. conductivity configuration of the anomalous zone and the host medium) as long as the forward modeling is also convergent. In this case, the Markov chain retains its 1-D character as described in Section 2, i.e. it has only one discrete variable which is the conductance of the thin-sheet.

\section{Inversion of Synthetic Data}

\subsection{Synthetic model and synthetic data}

In this study, synthetic thin-sheet models corresponding to a regional or large scale environment were used to generate synthetic data. The typical 1-D model consists of four layers having resistivities of 10 or $500 \mathrm{Ohm} . \mathrm{m}, 500 \mathrm{Ohm} . \mathrm{m}$, 1000 Ohm.m and 10 Ohm.m with thickness of 5, 40, 70 $\mathrm{km}$ respectively. The thin-sheet is the uppermost or surface layer and the resistivity contrast between anomalous zone and the normal host is 500 to $10 \mathrm{Ohm}$.m which correspond to a contrast in conductance of 10 to 500 Siemens (i.e. a resistive structure embedded in a conductive layer). For a conductive structure embedded in a resistive layer, resistivities are simply interchanged. In the subsequent paragraphs we denote the two cases by the anomalous zone, i.e. resistive anomaly and conductive anomaly. The thin-sheet containing the anomalous zone is divided into $10 \times 10$ cells, each has 30 $\mathrm{km}$ wide. Outside that zone the medium is 1-D as described above (Fig. 3).
At the center of each cell we calculated the electric and magnetic fields due to conductivity configuration in the synthetic model described above. The EM fields components are in the orthogonal coordinate system commonly adopted in the geomagnetic studies, i.e. $x$ positive to the North, $y$ positive to the East and $z$ positive downwards. The periods used (300, 1000, 3600 and 7200 seconds) and the layer discretization are such that the thin-sheet approximation holds. The complex transfer functions common to EM studies (i.e. impedance tensor and magnetic transfer function or induction vector) were then calculated according to the following well-known relations

$$
\begin{aligned}
& \left(\begin{array}{l}
E_{x} \\
E_{y}
\end{array}\right)=\left(\begin{array}{ll}
Z_{x x} & Z_{x y} \\
Z_{y x} & Z_{y y}
\end{array}\right)\left(\begin{array}{l}
H_{x} \\
H_{y}
\end{array}\right) \text { or } \mathbf{E}=\mathbf{Z} \cdot \mathbf{H}, \\
& H_{z}=T_{z x} H_{x}+T_{z y} H_{y} .
\end{aligned}
$$

A $10 \%$ Gaussian error was then added independently to real and imaginary parts of the transfer functions to simulate noise in the synthetic data. The noise corrupted complex impedance tensor and induction vector were used in all inversions. Apparent resistivity and phase calculated from components of the impedance tensor are commonly used for data presentation purposes. The relationship between the phase and the geometry of the structure is less obvious. Figure 4 shows only apparent resistivity maps of the principal components (i.e. $\rho_{x y}, \rho_{y x}$ ) corresponding to resistive and conductive anomaly for $T=1000 \mathrm{sec}$. It is also a common practice that the magnetic transfer function is presented as 
Table 1. Quasi-linearly discretized prior conductances (50 Siemens interval except for values lower than 50 Siemens) with their corresponding resistivity values and anomalous conductance relative to normal conductance for resistive and conductive anomalies.

\begin{tabular}{cccc}
\hline Conductance & Resistivity & \multicolumn{2}{c}{ Anomalous conductance (Siemens) } \\
\cline { 3 - 4 }$($ Siemens $)$ & $($ Ohm.m $)$ & Resistive anomaly & Conductive anomaly \\
\hline 1.0 & 5000.0 & -499.0 & -9.0 \\
10.0 & 500.0 & -490.0 & 0.0 \\
50.0 & 100.0 & -450.0 & 40.0 \\
100.0 & 50.0 & -400.0 & 90.0 \\
150.0 & 33.3 & -350.0 & 140.0 \\
200.0 & 25.0 & -300.0 & 190.0 \\
250.0 & 20.0 & -250.0 & 240.0 \\
300.0 & 16.7 & -200.0 & 290.0 \\
350.0 & 14.3 & -150.0 & 340.0 \\
400.0 & 12.5 & -100.0 & 390.0 \\
450.0 & 11.1 & -50.0 & 440.0 \\
500.0 & 10.0 & 0.0 & 490.0 \\
550.0 & 9.1 & 50.0 & 540.0 \\
600.0 & 8.3 & 100.0 & 590.0 \\
\hline
\end{tabular}

Table 2. Quasi-logarithmically discretized prior conductances with their corresponding resistivity values and anomalous conductance relative to normal conductance for resistive and conductive anomalies.

\begin{tabular}{cccc}
\hline Conductance & Resistivity & \multicolumn{2}{c}{ Anomalous conductance (Siemens) } \\
\cline { 3 - 4 }$($ Siemens $)$ & $($ Ohm.m $)$ & Resistive anomaly & Conductive anomaly \\
\hline 1.0 & 5000.0 & -499.0 & -9.0 \\
2.0 & 2500.0 & -498.0 & -8.0 \\
5.0 & 1000.0 & -495.0 & -5.0 \\
10.0 & 500.0 & -490.0 & 0.0 \\
20.0 & 250.0 & -480.0 & 10.0 \\
50.0 & 100.0 & -450.0 & 40.0 \\
100.0 & 50.0 & -400.0 & 90.0 \\
200.0 & 25.0 & -300.0 & 190.0 \\
500.0 & 10.0 & 0.0 & 490.0 \\
1000.0 & 5.0 & 500.0 & 990.0 \\
2000.0 & 2.5 & 1500.0 & 1990.0 \\
5000.0 & 1.0 & 4500.0 & 4990.0 \\
\hline
\end{tabular}

real and imaginary induction arrows according to the following convention

$$
\begin{aligned}
& \bar{V}_{\operatorname{Re}}=-\operatorname{Re}\left(T_{z x}\right) \hat{x}-\operatorname{Re}\left(T_{z y}\right) \hat{y}, \\
& \bar{V}_{\operatorname{Im}}=\operatorname{Im}\left(T_{z x}\right) \hat{x}+\operatorname{Im}\left(T_{z y}\right) \hat{y} .
\end{aligned}
$$

where $\hat{x}$ and $\hat{y}$ are unitary vector in the $x$ - and $y$-axes respectively. Note that the direction of the real induction vector is inverted such that it conforms to the Parkinsons induction arrows. With this convention, the real induction arrows point towards the conductive medium (Fig. 5). From Figs. 4 and 5 we can observe relative sensitivities of different impedance tensor components and also different types of data (apparent resistivity or real and imaginary parts of the induction arrows) related to the form of the anomaly. This may indicate which characteristic feature of the model than can be inferred from these different type of data in the qualitative interpretation. For example, it is well known that apparent resistivity map reveals more clearly conductivity contrast perpendicular to the electric field direction (TM mode) and so forth.

\subsection{Prior model}

To simplify the problem, we consider that model parameters defining the stratified or 1-D medium is known and equal to the model parameters used to create the synthetic data. In the case of real data inversion we need this information as a supplementary prior information which may be predominant in the inversion process. We used a uni- 


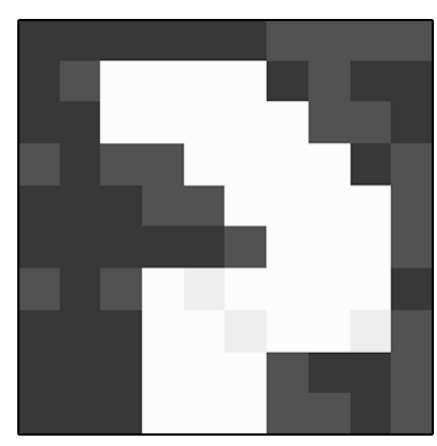

(a)

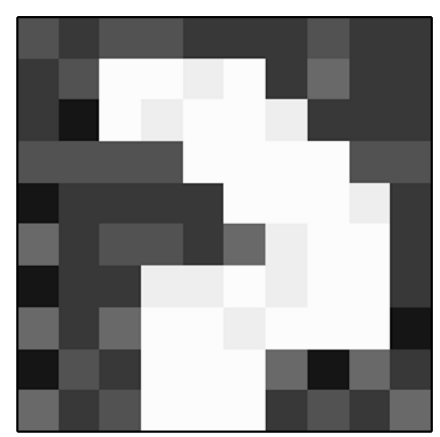

(c)

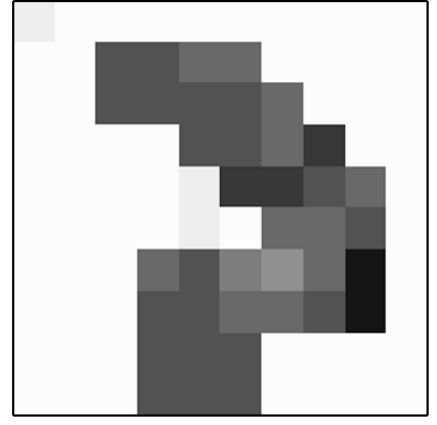

(b)

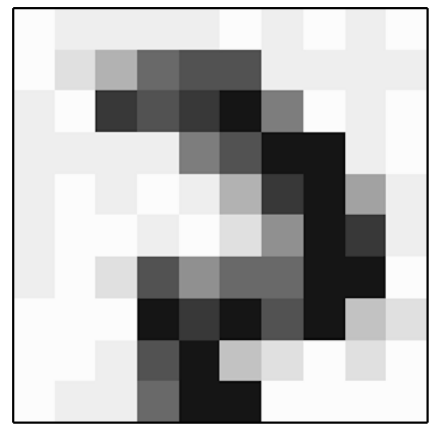

(d)

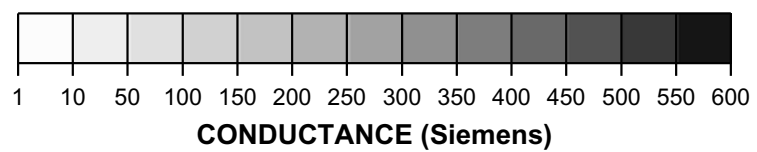

Fig. 6. Conductance map obtained from inversion using linear discretization of prior conductance values: inversion of impedance tensor for (a) resistive and (b) conductive anomaly, inversion of induction vector for (c) resistive and (d) conductive anomaly.

form pdf for prior conductances in a given interval covering the anomalous and normal conductance values. In this interval, a set of discrete conductance values were used as prior or possible values for the model parameter (conductance of each cell). The discretization interval of possible conductance values may substantially dictate the inversion results. Therefore, we used two kinds of discretization, i.e. quasi-linear and quasi-logarithmic discretizations (see Tables 1 and 2) to evaluate their influence on the sensitivity of the inversion algorithm and also on the marginal posterior probability of the model parameters. Note that in Tables 1 and 2 anomalous conductance signifies conductance difference of a cell or a block relative to the conductance of the host or normal thin-layer. In subsequent paragraphs, quasilinear and quasi-logarithmic discretization of prior conductance will be termed simply linear and logarithmic prior conductance values.

\subsection{Results}

A number of inversions were performed using both linear and logarithmic prior conductance values with the tensor impedance and induction vector as the data. Preliminary tests showed that the convergence was obtained after 5 to 10 iterations after which posterior values (marginal pdf and model parameters) oscillate around their optimum values. Inversions are systematically effected up to 20 itera- tions and results of the last 15 iterations were averaged. Figures 6 and 7 show the inversion results using linear and logarithmic prior conductance values respectively. The results are presented as maps representing conductance distribution in the thin-sheet. In general, the true structure can be resolved fairly well, except in the case of inversion of induction vector for conductive anomaly (Figs. 6(d) and 7(d)). In the latter, the result probably stems from the fact that a conductive anomaly confined in a resistive host produces a low magnitude anomaly, especially in terms of induction vector (Menvielle et al., 1982). Therefore, poor resolution of this type of anomaly is mainly related to the type of data since the same conductive anomaly is more clearly resolved from inversion of the impedance tensor (Figs. 6(b) and 7(b)).

A detailed observation of the inversion results reveals characteristics of the inversion method which may be attributed to differences in linear and logarithmic prior conductance values. Resistive zones, whether they are in the host medium or in the anomalous zone, are well resolved from inversions using linear prior conductance values. In these resistive zones, conductance variations are less such that they appear as a good enitity (Figs. 6(a), (b) and (c)). In contrast, conductive zones are well resolved from inversion using logarithmic prior conductance values, especially in Fig. 7(a) and in a lesser extent in Figs. 7(b) and (c). Thus, 


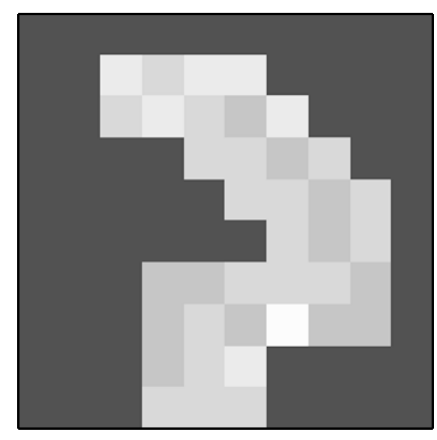

(a)

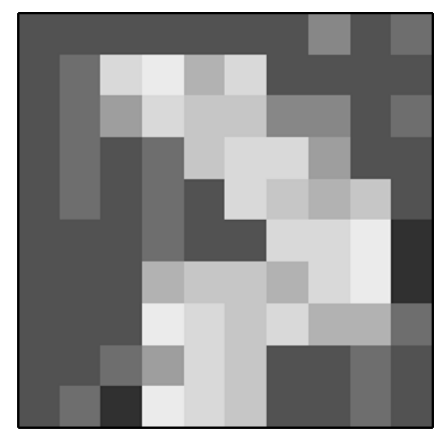

(c)

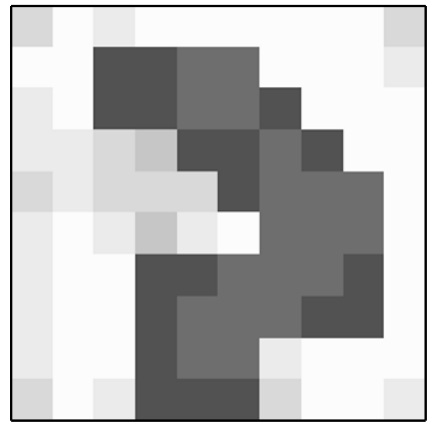

(b)

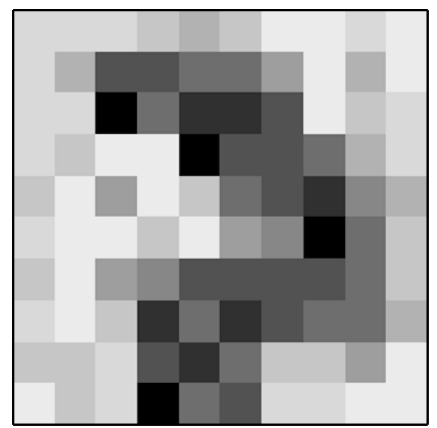

(d)

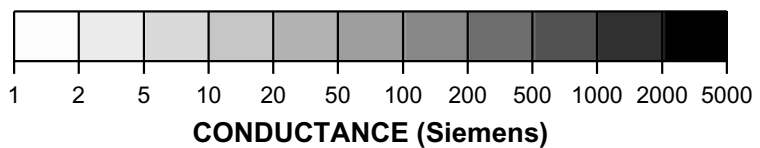

Fig. 7. Conductance map obtained from inversion using logarithmic discretization of prior conductance values: inversion of impedance tensor for (a) resistive and (b) conductive anomaly, inversion of induction vector for (c) resistive and (d) conductive anomaly.

different discretization of prior conductance leads to different effect on the resolution of resistive and conductive zones. In case of linear prior conductance values, the inversion algorithm is able to differentiate $1,10,50$ and 100 Siemens and to choose the true value (10 Siemens) for resistive zones. For conductive zones, the inversion method has difficulty to distinguish conductance values between 350 and 600 Siemens in 50 Siemens interval. In case of logarithmic prior conductance values, conductive zones appears to be better resolved due to a large difference in prior conductance values for the conductive interval $(100,200,500$ and 1000 Siemens).

By assuming that the thickness of the thin-sheet is known, linear prior conductance values for resistive zones are obviously different expressed in terms of resistivity, i.e. 5000, $500,100,50 \mathrm{Ohm} . \mathrm{m}$. For conductive zones, linear prior conductance corresponds to resistivities between 8 to $15 \mathrm{Ohm} . \mathrm{m}$ which are imperceptible in the inversion. In this interval, a conductive zone can be characterized by any resistivity value. The same explanations are also valid for logarithmic prior conductance values (see Table 2 ).

The transition probability of the Markov chain at the last (20-th) iteration supports more clearly the facts described in the above paragraph. The transition probability is theoretically convergent to the marginal posterior probability of the model parameters, although the latter is generally better estimated using empirical average of the transition probabilities (Grandis et al., 1999; Schott et al., 1999). In the following only results from inversion of the impedance tensor are presented. Figure 8 presents the transition probability of each model parameter at the last iteration for linear prior conductance values. The probability is represented as histogram plotted at each cell where the horizontal axis is the conductance scale. A zoom of the transition probability for two adjacent cells indicated by arrows is also shown in Fig. 8. The same set of figures for logarithmic prior conductance values are presented in Fig. 9. A well resolved model parameter is characterized by a nearly single bar at or around the true conductance value (i.e. resistive at the left side of each cell). In contrast, a less well resolved model parameter may be identified from nearly uniform small bars around the true conductance value (i.e. conductive at the right side of each cell). Similar results were also obtained from inversion of the induction vector data.

\section{Conclusion}

In this paper we described the integration of an existing thin-sheet forward modeling scheme into a stochastic inversion method based on MCMC algorithm. Empirical study on convergence of the forward modeling has permitted to 

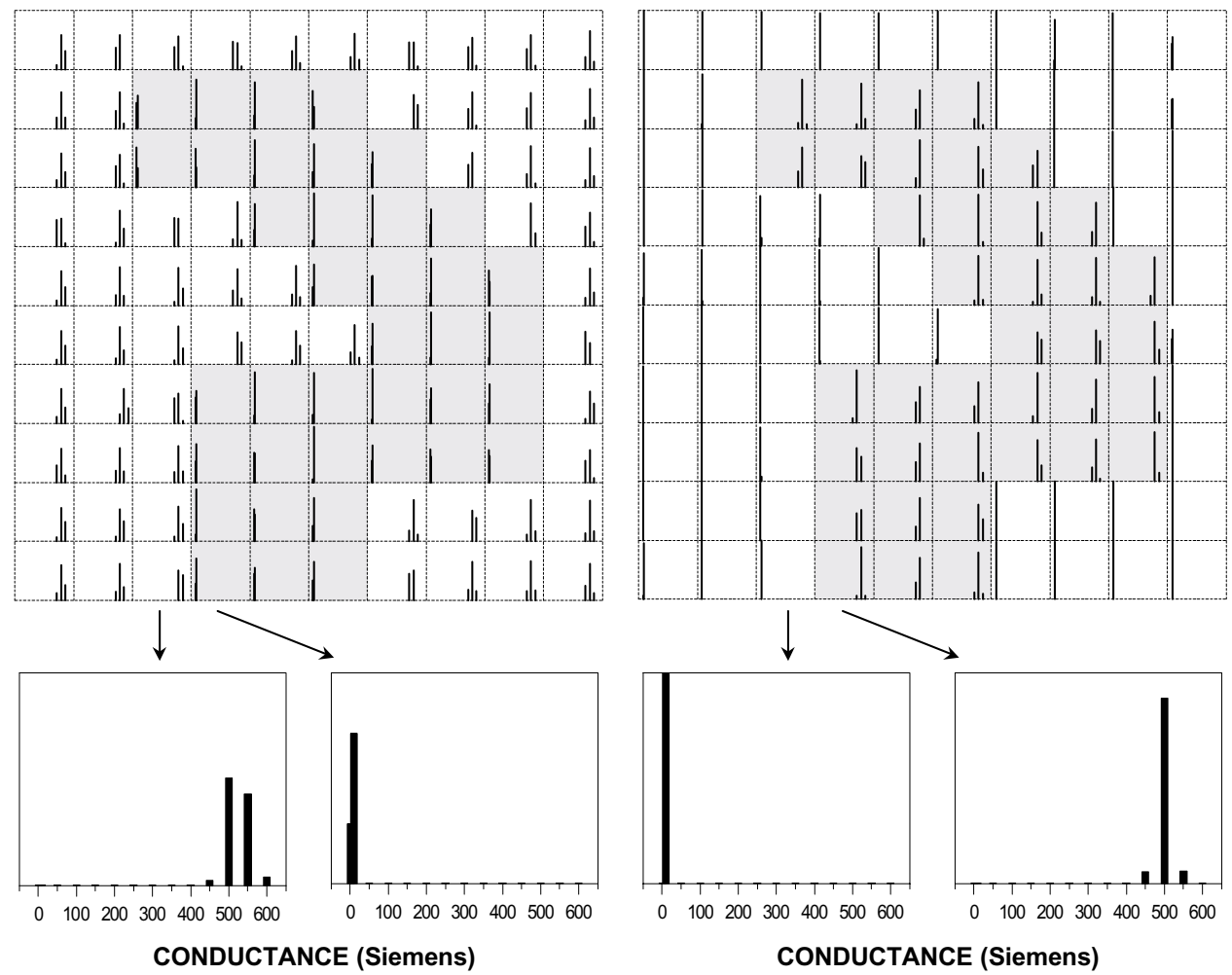

Fig. 8. Histogram representing transition probability at the last (20-th) iteration of each cell for linear discretization of prior conductance values: inversion of impedance tensor for resistive (left) and conductive anomaly (right). A zoom of the transition probability for two adjacent cells indicated by arrows is shown below each figure.

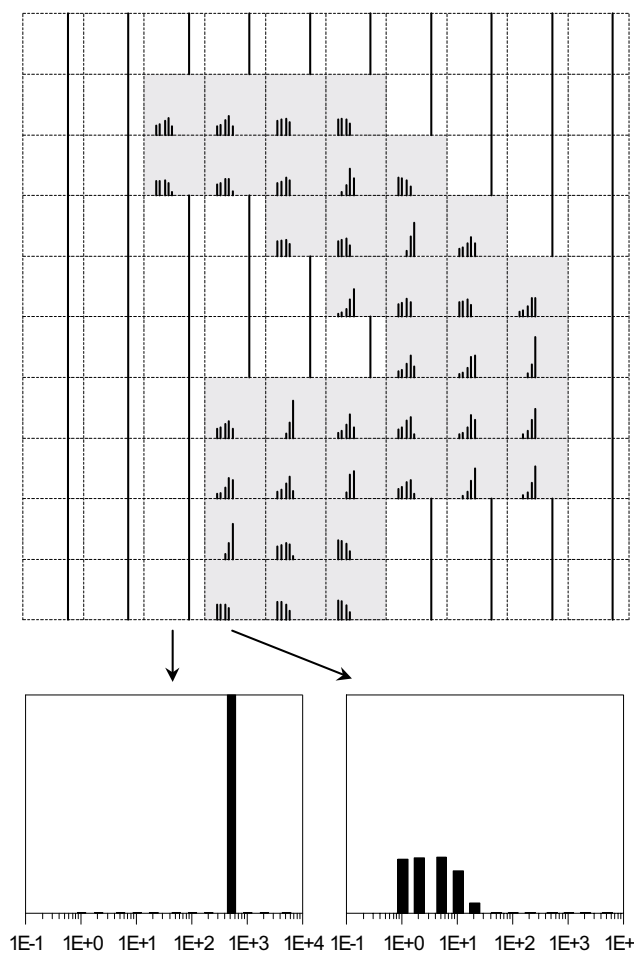

CONDUCTANCE (Siemens)

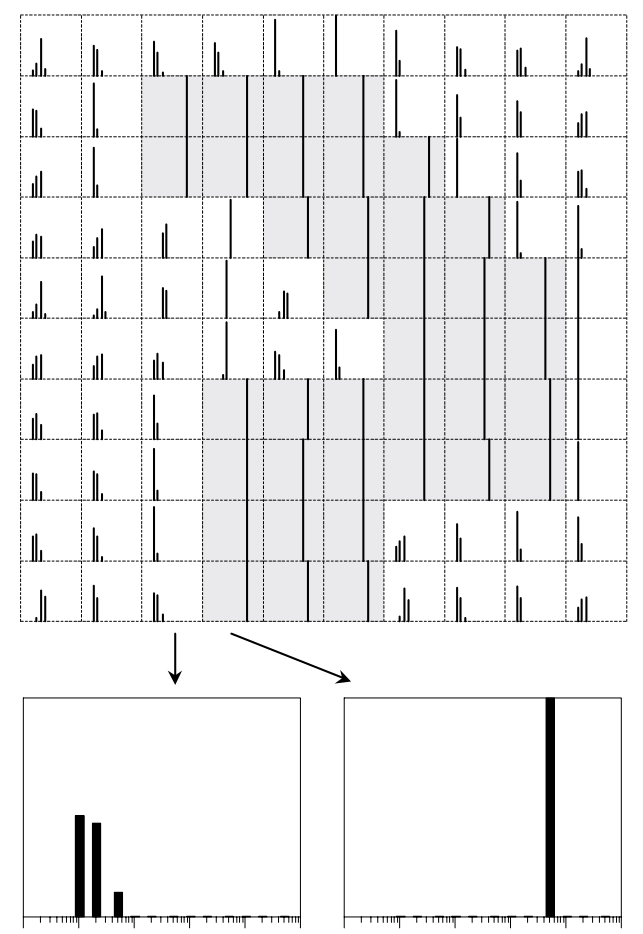

CONDUCTANCE (Siemens)

Fig. 9. Same as Fig. 8 but for logarithmic discretization of prior conductance values. 
recognize that approximate estimation in the calculation of the Markov chain's transition probability was inadequate, especially for conductive anomalies. This leads to an idea of incorporating more Gauss-Seidell iterations in the calculation of the forward modeling such that more accurate estimate of the transition probability is obtained. The modified inversion algorithm is thus applicable to invert data corresponding to both resistive and conductive anomaly as well. From theoretical point of view, the modification also preserves one-dimensional Markov chain for which mathemartical proof of convergence has been established (Grandis, 1994, 2002).

The effectiveness of the algorithm was tested by performing inversions of synthetic data in the form of impedance tensor and induction vector. In general, synthetic models containing resistive and conductive anomalies are fairly well resolved. Best results were obtained from inversion of the MT impedance tensor data suggesting that these kind of data provide more information on the conductivity distribution in the thin-sheet. Different discretization of prior conductance values revealed that, for a given thickness of the thinsheet, the inversion algorithm is more sensitive to resistivity value of the thin-sheet. Thus, in discretizing prior conductance we have to consider the corresponding resistivity values such that the inversion can resolve both resistive and conductive anomalies equally well with a reasonable resolution. This implies that the thickness of the thin-sheet must be relatively well known a priori. Provided that this a priori information is known, a possible strategy in applying the inversion method to real data consists of (i) inversion using a coarse prior conductance values in a large interval to identify predominant anomalies, and (ii) inversion using a finer discretization of prior conductance around these predominant anomalies. Such strategy is necessary to obtain a good model resolution while keeping reasonable computational time by using a relatively limited number of prior conductance values. Note that the number of prior conductance values corresponds to the number of forward modeling in each step of the algorithm, i.e. calculation of the transition probability used to update a model parameter.

The proposed inversion method belongs to global search and gradient-free optimization methods which can effectively overcome difficulties of local search or linearized approach of strongy non-linear problems. The MCMC algorithm is, in general, computer intensive and slow since a large number of forward modeling has to be effected to resolve the inverse problem. However, more informative solution in terms of marginal posterior pdf of the model parameters represent invaluable information in assessing the uncertainty and, to some extent, the non-uniqueness of the solution (Sen and Stoffa, 1996). For cases in which the pdf of the model parameters is not Gaussian nor unimodal (which is the case for most non-linear problems), standard estimators (i.e. mean and variance) would be severely biased and even meaningless in the worst cases (Tarits et al., 1994). It is then important to base our interpretation of the inverted models directly using the marginal posterior pdf of the model parameters.

Acknowledgments. The authors greatly acknowledge Pascal Tarits and Virginie Jouanne for fruitful discussions during the preparation of this paper.

\section{References}

Grandis, H., Imagerie electromagnetique Bayesienne par la simulation d'une chaîne de Markov, Ph.D. thesis, Université Paris 7, 278 pp., 1994

Grandis, H., Application of magnetotelluric (MT) method in mapping basement structures: Example from Rhine-Saone Transform Zone, France, Indonesian Mining Journal, 3, 16-25, 1997.

Grandis, H., M. Menvielle, and M. Roussignol, Bayesian inversion with Markov chains-I. The magnetotelluric one-dimensional case, Geophys. J. Inter, 138, 757-768, 1999.

Grandis, H., M. Menvielle, and M. Roussignol, Monte Carlo Markov Chains for non-linear inverse problems: an algorithm, Mathematical Geology, 2002 (submitted).

Heerman, D. W., Computer simulation methods in theoretical physics, Springer-Verlag, Berlin, 1990.

Jackson, D. D. and M. Matsu'ura, A bayesian approach to nonlinear inversion, J. Geophys. Res., 90(B1), 581-591, 1985

Jain, M. K., S. R. K. Iyengar, and R. K. Jain, Numerical methods for scientific and engineering computation, Wiley Eastern, 1987.

Jouanne, V., Application des techniques statistiques bayesiennes à l'inversion de données électromagnétiques, Ph.D. thesis, Université Paris 7 , 1991.

McKirdy, D. McA., J. T. Weaver, and T. W. Dawson, Induction in a thin sheet of variable conductance at the surface of a stratified earth-II: Three-dimensional theory, Geophys. J. Roy. astr. Soc., 80, 177-194, 1985.

Menvielle, M., J. C. Rossignol, and P. Tarits, The coast effect in terms of deviated electric currents: a numerical study, Phys. Earth Planet. Inter, 28, 118-128, 1982.

Robert, C., L'analyse Statistique Bayesienne, 393 pp., Economica, Paris, 1992.

Robert, C., Méthodes de Monte Carlo par Chaînes de Markov, 393 pp., Economica, Paris, 1996.

Rothman, D. H., Automatic estimation of large residual statics corrections, Geophysics, 51, 332-346, 1986.

Roussignol, M., V. Jouanne, M. Menvielle, and P. Tarits, Bayesian electromagnetic imaging, in Computer Intensive Methods, edited by W. Hardle and L. Siman, pp. 85-97, Physical Verlag, 1993.

Schott, J.-J., M. Roussignol, M. Menvielle, and F. R. Nomenjahanary, Bayesian inversion with Markov chains-II. The one-dimensional DC multilayer case, Geophys. J. Inter., 138, 769-783, 1999.

Sen, M. K. and P. L. Stoffa, Bayesian inference, Gibbs' sampler and uncertainty estimation in geophysical inversion, Geophys. Prosp., 44, 313350, 1996.

Tarits, P., V. Jouanne, M. Menvielle, and M. Roussignol, Bayesian statistics of non-linear inverse problems: examples of the magnetotelluric 1-D inverse problem, Geophys. J. Inter., 119, 353-368, 1994.

Vasseur, G. and P. Weidelt, Bimodal electromagnetic induction in nonuniform thin sheets with an application to the northern Pyrenean induction anomaly, Geophys. J. Roy. astr. Soc., 51, 669-690, 1977.

Wang, L. J. and F. E. M. Lilley, Inversion of magnetometer array data by thin-sheet modeling, Geophys. J. Inter., 137, 128-138, 1999.

H. Grandis (e-mail: grandis@geoph.itb.ac.id), M. Menvielle, and M. Roussignol 